\title{
Vitamin D Deficiency among Depressed Women
}

\author{
Rawa Al Ameri* \\ Family Medicine Specialist, Al-Mustansiriyah University, Iraq
}

*Corresponding author: Rawa Al-Ameri, Family Medicine Specialist, AlMustansiriyah University, Iraq, Email: dr_rj1983@yahoo.com

\section{Research Article \\ Volume 3 Issue 1}

Received Date: December 16, 2019

Published Date: January 09, 2020

DOI: $10.23880 /$ jqhe- 16000148

\section{Abstract}

Introduction: Iraq is a sunny country so the risk of vitamin d deficiency is underestimated. Vitamin d deficiency is used to be related to rickets in children and osteomalacia in adults, so other symptoms are somewhat undistinguished. In recent years, many patients presented to primary health care with symptoms of fatigue, tiredness, bone and back pain, depression, hair loss, and muscle pain, as these symptoms raise the suspicion that vitamin $\mathrm{d}$ deficiency is becoming a concerning issue. Inadequate vitamin $\mathrm{d}$ level is an international topic now a day. Cases of depression are so common in daily routine work in primary health care, especially in women. In recent years marked attention is applied to support the depressed patients in primary health care.

Objectives:-Assessment of $25(\mathrm{OH})$ D levels in depressed women.-Assessment of vitamin D deficiency according to age groups and pregnancy state.

Methods and Results: This is a cross-sectional study, a comprehensive sampling technique, included 108 women, above fifteen years old, presented to primary health care with symptoms of depression. The women have been sent for vitamin $\mathrm{d}$ level assessment. The results showed Vitamin d deficiency in a 100\% of participated depressed women, including 40 pregnant. Serum vitamin d level is assessed by using $25(\mathrm{OH})$ vitamin D ELISA kit, which is available in most labs in the area of the primary health care center. The results showed $100 \%$ inadequate level of serum $25(\mathrm{OH}) \mathrm{D}, 79.1 \%$ of depressed women had deficient state while $20.9 \%$ of women had insufficient titer.

Discussion and Conclusion: Family physician has an important role in the detection of psychological cases including depression and offering a plan for management and follow up. Randomized trials suggest that vitamin D supplementation can improve mood and health status in women. The current study gives a conclusion that vitamin d deficiency could be associated with depression in women.

Keywords: Vitamin D Deficiency; Primary Health Care; Fatigue

\section{Introduction}

Iraq is a sunny country so researchers consider vitamin d deficiency concept is far away. Since vitamin d deficiency is used to be related to rickets in children and osteomalacia in adults, so other symptoms are somewhat undistinguished [1].

In recent years, many patients presented to primary health care with symptoms of fatigue, tiredness, bone and 


\section{Journal of Quality in Health Care \& Economics}

back pain, depression, hair loss, and muscle pain, as these symptoms raise the suspicion that vitamin d deficiency is becoming a concerning issue [1-3]. Inadequate vitamin $d$ level is an international topic now a day [1].

Cases of depression are so common in daily routine work in primary health care, especially in women. In recent years a growing attention is applied for caring the depressed cases due to the stressful events that arise in the country due to civilized wars and fight issue, as a psychological support unit is created in each primary health care with trained professional staff, but the numbers of depressed cases continue to rise despite the settlement and peace measures.

Besides, other associated symptoms as fatigue and hair loss have emerged which gave a clue that many of these depressed cases could be associated with inadequate vitamin d level [4].

"Depressive disorders are expressed as loss of interest or pleasure, sadness, feelings of guilt or low self-worth, disturbed sleep or appetite, feelings of tiredness, and poor concentration." It can cause impairment of the ability to function at work or school or cope with daily life. In severe case, may lead to suicide.

Depressive disorders include two main sub-categories: major depressive disorder and dysthymia. The depressive episode involves symptoms such as depressed mood, loss of interest and enjoyment, and decreased energy while dysthymia is a persistent or chronic form of mild depression [5].

There is adequate evidence for a positive association between vitamin $\mathrm{D}$ deficiency and depression as many studies showed this relation. Parker GB, et al. [6] offers a review explains the role of vitamin $\mathrm{D}$ supplementation in treatment for depression.

In the same way, a meta-analysis of randomized controlled trials done by Usha Gowda M.H.N., et.al exhibited the efficacy of vitamin D supplementation on depression which involved individuals aged $\geq 18 \mathrm{y}$ diagnosed with depressive disorder.

Another study is done by Penckofer S, et al. [7] displayed the effect of vitamin $\mathrm{D}$ supplementation on improving mood and health status in women with type 2 diabetes mellitus as it confirmed the role sunshine on mental and physical health.

Observational data revealed that low vitamin $\mathrm{D}$ is related to adverse brain and behavior outcomes in animal models so this raises the suggestion that Vitamin D supplementation could be related to better later-life mood and Preventing late-life depression [8].

The most important category of society is a pregnant woman; a healthy pregnant will form a healthy child and healthy adult and in turn, a healthy community will be formed with better consumption of health resources. Pregnant women require special care with attention on a healthy diet to avoid deficiency of important vitamins and minerals.

Many studies presented the importance of vitamin $d$ and calcium to pregnant by its beneficial effect on fetal bone development [9]. In addition to the studies that linked their deficiency with toxemia in pregnancy [10].

New theories are updated about gestational vitamin D deficiency as it is associated with altered brain development; this common exposure is a candidate modifiable risk factor for autism-related traits. A study was done by Vinkhuyzen AAE, et al. [11] connected between lower mid-gestation 25-hydroxyvitamin D (250HD) concentrations and higher scores on the Attention Switching subscale of the Autism-Spectrum Quotient.

Neonatal vitamin D deficiency has been previously associated with an increased risk of schizophrenia. The active form of vitamin D $(1,25$ dihydroxy vitamin D contribute to the neurodevelopmental disorders by its impact on the function of voltage-gated calcium channels [12]. In addition, vitamin $\mathrm{D}$ has been linked to other neurobiological features associated with autism as tryptophan hydroxylase which is a key enzyme in the production of serotonin and impairment of GABA synthesis pathway [12-14].

In recent years, rates of depression among pregnant women are increased dramatically as the estimated rates of depression during pregnancy are $7-15 \%$ in economically developed countries $[15,16]$.

Women are about twice as likely as men to develop major depression as hormone levels fluctuate wildly. They also have higher rates of seasonal affective disorder, depressive symptoms in bipolar disorder, and dysthymia. Studies found a higher prevalence in girls starting at age $11[17,18]$.

Paul R Albert, et al. presented a study in 2015 revealed that female: male ratio of global disability from major depression remained unchanged at 1.7:1. 
Globally, depression is more than twice as prevalent in young women as men particularly in ages 14-25 years, but this ratio decreases with age [19].

Other risk factors that could be associated with depression prevalence among women; life stressors, previous episodes of depression, postpartum depression or PMS, lack of social support, unwanted pregnancy, miscarriage, and infertility [17-19].

Scientist over decades tried hard to improve the management and follow up of depressed patient as a study done by Nayereh Khoraminya, et al. [20] gave a result that adding vitamin D supplement to fluoxetine was superior to fluoxetine alone in controlling depressive symptoms.

Similarly, a study offered by Mozaffari Khosravi, et al. [21] as injection of vitamin $d$ to depressed patients improved the depression state.

\section{Objectives:}

- Assessment of $25(\mathrm{OH})$ D levels in depressed women.

- Assessment of vitamin D deficiency according to age groups and pregnancy state.

\section{Subjects and Methods}

\section{Study Design}

This is a cross-sectional study, a comprehensive sampling technique, included 108 women, above fifteen years old, presented to primary health care with symptoms of depression. The women have been sent for vitamin $d$ level assessment.

Settings: This study was conducted in the Al-Dora Primary Healthcare Center for Family Health/Al-Karkh Health Directorate in Baghdad/Iraq.

> Sampling: The study was adopted by assessing serum $25(\mathrm{OH})$ D levels in all women above fifteen years old presented to primary health care with symptoms of depression for three months, between $1 / 5 / 2019$ to $1 / 8 / 2019$.

$>$ Tools and Instruments: Serum vitamin d level is assessed by using $25(\mathrm{OH})$ vitamin D ELISA kit, which is available in most labs in the area of the primary health care center.

> Administrative Approvals: Official approval is obtained from managers of Al-Dora primary Health care Center for Family Health, Al-Dora Primary Health care Sector, and Al-Karkh Health Directorate.

$>$ Data Collection: The sample included the women above fifteen years old that were presented with depressed symptoms, for three months, between $1 / 5 / 2019$ to $1 / 8 / 2019$. The study included 108 women. Serum vitamin d level is assessed by using 25(OH) vitamin D ELISA kit. Using the RDA-associated serum concentrations of vitamin $D$ to judge the results. Considering $<12.5 \mathrm{ng}$ per milliliter is deficient while $(12-19) \mathrm{ng} / \mathrm{ml}$ is insufficient.

\section{Data Management and Statistical Analysis}

Each woman assigned a serial identification number. The data were reviewed, cleaned with double-check entry into the computer using Statistical Package for Social Sciences (SPSS) version 18; then, it was coded. The data were presented as frequency, proportion, and percentage. Frequency tables and bar charts were used for data clarification. The participant women were subdivided according to their age group in addition to the pregnancy state.

\section{Results}

Out of 108 depressed women were sent to the lab for serum 25(OH) D, 91 women returned with the results Tables 1 \& 2, Figures 1-3.

\begin{tabular}{|c|c|}
\hline Total & $\mathbf{1 0 8}$ \\
\hline Respondents & 91 \\
\hline Response rate & $84.30 \%$ \\
\hline
\end{tabular}

Table1: Response rate in involved women.

\begin{tabular}{|c|c|c|c|c|c|}
\hline Age groups (years) & No. & $\mathbf{\%}$ & No. of pregnant women & \% from Total & \% from Age \\
\hline$<20$ & 10 & $11.00 \%$ & 7 & $17.50 \%$ & $70.00 \%$ \\
\hline $20-29$ & 33 & $36.30 \%$ & 24 & $60.00 \%$ & $72.70 \%$ \\
\hline $30-39$ & 18 & $19.80 \%$ & 7 & $17.50 \%$ & $38.90 \%$ \\
\hline $40-49$ & 16 & $17.60 \%$ & 2 & $5.00 \%$ & $12.50 \%$ \\
\hline$>=50$ & 14 & $15.40 \%$ & 0 & $0.00 \%$ & $0.00 \%$ \\
\hline Total & 91 & $100.00 \%$ & 40 & $100.00 \%$ & $44.00 \%$ \\
\hline
\end{tabular}

Table2: Age groups and No. of pregnant women. 
The results showed Vitamin d deficiency in $100 \%$ of participated depressed women, including 40 pregnant.

The women aged (20-29) years were more dominant in the study.

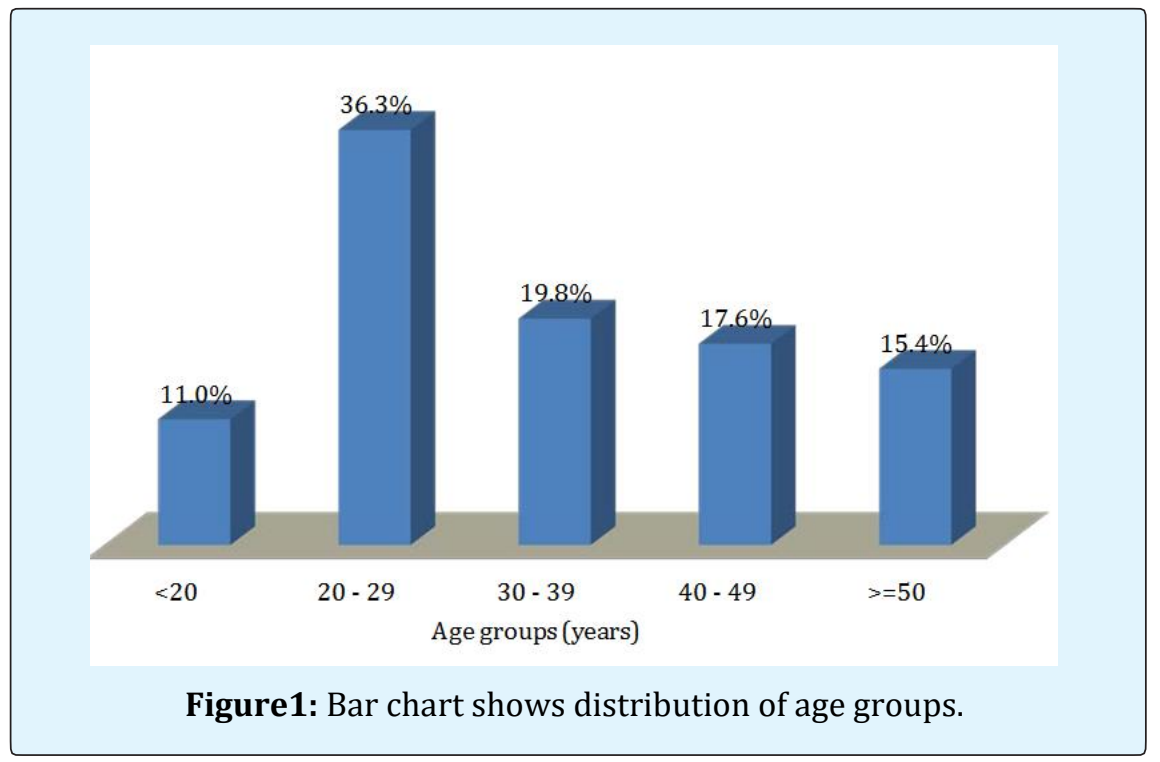

The highest number of pregnant women were more in age (20-29) years in the study, followed by the age group $<20$ years.

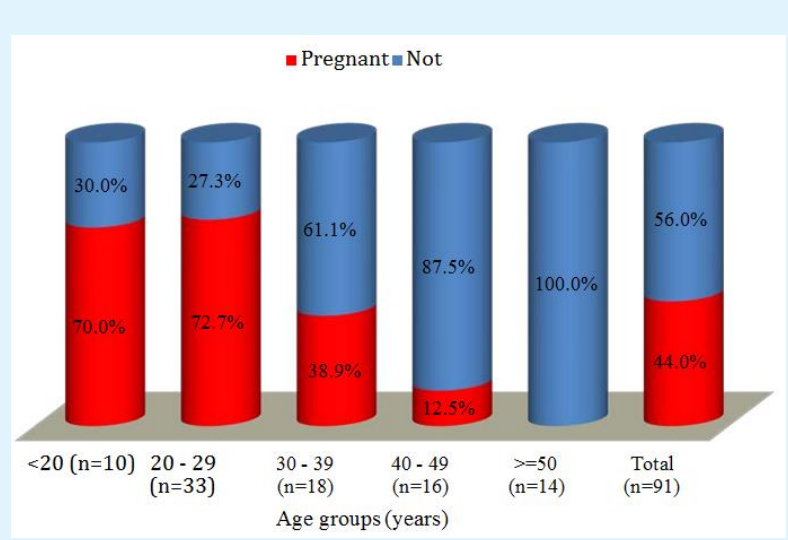

Figure2: The bar chart shows the distribution of pregnancy state according to age groups.

The results showed $100 \%$ inadequate level of serum $25(\mathrm{OH}) \mathrm{D}, 79.1 \%$ of depressed women had deficient state while $20.9 \%$ of women had insufficient titer, considering
$20 \mathrm{ng} / \mathrm{ml}$ is a cutoff point as $12-19 \mathrm{ng} / \mathrm{ml}$ is deficient level and $<12 \mathrm{ng} / \mathrm{ml}$ is insufficient level of vitamin $\mathrm{d}$ (Table 3) [22].

\begin{tabular}{|c|c|c|}
\hline Vitamin D titer and status & $\mathbf{N}$ & $\mathbf{\%}$ \\
\hline$<12 \mathrm{ng} / \mathrm{ml}$ (Deficient) & 72 & $79.10 \%$ \\
\hline $12-19 \mathrm{ng} / \mathrm{ml}$ (Insufficient) & 19 & $20.90 \%$ \\
\hline Total & 91 & $100.00 \%$ \\
\hline
\end{tabular}

Table 3: The percentage of the deficient and insufficient state of vitamin D. 


\section{Journal of Quality in Health Care \& Economics}

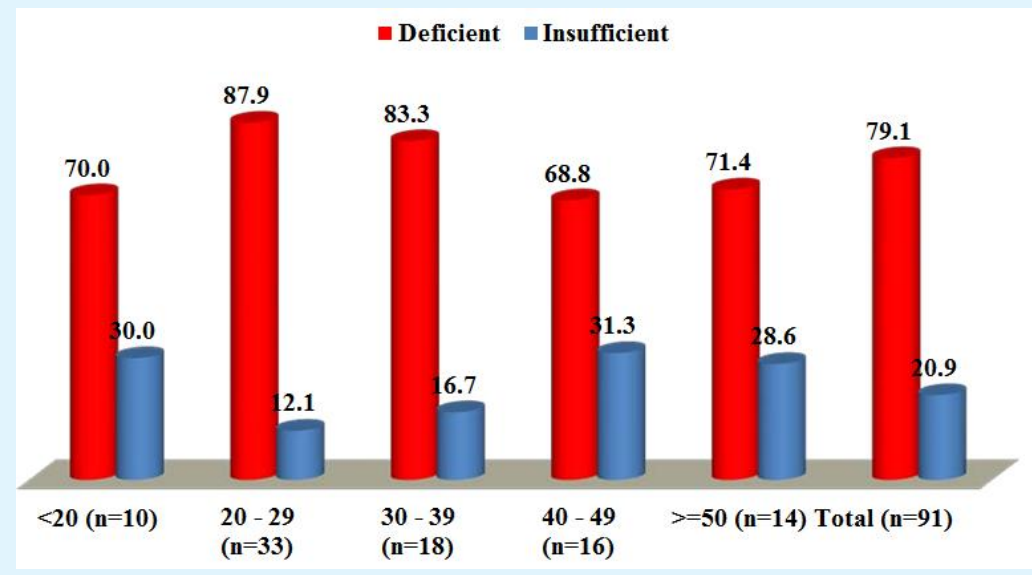

Figure 3: The bar chart shows the deficient and insufficient state of vitamin D level according to age groups.

The young women had the highest percentage of the deficient state of vitamin D; (20-29) years followed by (30-39) years, while women aged (40-49) years had the highest percentage of the insufficient state. On the other hand, $100 \%$ of pregnant presented in a deficient state.

\section{Discussions}

Family physician has an important role in detection of psychological cases including depression and offering a plan for management and follow up, in addition to their role in Counseling which is a distinct from psychotherapy and" it is that form of activity in which the physician engages in an educational dialogue with the patient, on an individual or group basis, wherein the goal of the physician and patient is to become aware of the patient's problems or situation and the modalities for prevention or treatment", besides their role in supporting the patients and their families through emotional and physical illness with understanding, empathy and careful advice. This will make the primary health care not just an office offering medications but it is a comprehensive service takes-care the patients and their families [23].

The study revealed that $100 \%$ of participated depressed women had vitamin d deficiency; this could be a surprising result in a sunny country like Iraq. Actually, the hot weather and intense sunshine could force people to stay at home instead of walking on a sunny day; even they added tinted glass to prevent the sunshine from entering their houses. Clothes in women have an effect, too, as women used to wear completely covered styles due to cultural and religious beliefs.
This study stated that $79.1 \%$ of depressed women had deficient state while $20.9 \%$ of women had insufficient titer, which is fairly similar to a recent statistics in the United Kingdom showed that $74 \%$ of people are in the lower quarter of the range or below for their vitamin D levels which mean they are either deficient or insufficient level, with $27 \%$ of people actually falling below the normal parameters [24]. These numbers pose an issue, despite the extreme difference in weather; Iraq almost has vitamin d deficiency at the same levels.

The current study showed the highest percentage of the deficient state of vitamin D in age group ; $(20-29)$ years followed by age group (30-39) years; $87.9 \%$ and $83.3 \%$, while women aged (40-49) years had the highest percentage of the insufficient state; $31.3 \%$. On the other hand, the previous data revealed that those aged 25-34 and $45-54$ both have the lowest levels at $76 \%$ taking in regards the difference in sampling technique in both studies [24].

In Older age groups $>50$ years; $71.4 \%$ of them showed deficient level and $28.6 \%$ with the insufficient state is compared to a study done in Elderly Day Hospital, Dublin, which revealed that $30 \%$ of elderly were deficient and $49 \%$ of patients had insufficient levels with regarding of different sampling technique [25].

Elderly patients require special attention so that the primary health care offers support and care to this category. They are more vulnerable to vitamin $d$ deficiency with their complicated consequences as the risk of falls and trauma due to decrease their ability with advanced age to move and walk around in sunny weather. This, in turn, will increase their isolation with a long stay 


\section{Journal of Quality in Health Care \& Economics}

at homes predisposing to depression that could be associated with vitamin $\mathrm{d}$ deficiency.

The current study presented that $44.0 \%$ of the sample were pregnant women with $100 \%$ of the deficient level of vitamin d. Depression in pregnancy always claimed to hormonal variation but vitamin $d$ deficiency added other factors. Studies in this aspect emerged in recent years showing the effect of hypovitaminosis not only to the mother but also on their babies as it is associated with an increase of the risk of autism, schizophrenia and other neurodevelopmental disorders as impairment on a range of cognitive outcomes related to language, motor development and general intelligence of the child $[11,26]$.

Studies suggest the possibility of vitamin D to play an autocrine or paracrine action in the brain that may affect the pathophysiology of depression. Vitamin D could act as a neuroactive/neurosteroid hormone that is involved in key functions such as neuroprotection, neuroimmunomodulation, brain development, and regular brain function $[27,28]$.

Furthermore, there is emerging evidence of possible neuroprotective roles that vitamin D may play through its effects on inflammation as data suggest that the upregulation of proinflammatory cytokines in the brain may be associated with depression. Vitamin D may act as a modulator in the association between depression, and inflammatory response through its effect on the immune system [29,30].

In the Middle East and North Africa region, few recent RCTs that attempted to determine the optimal regimen of vitamin D replacement in healthy pregnant women as hypovitaminosis is prevalent worldwide, especially in developing countries [31].

Furthermore, decreased vitamin d during pregnancy could be associated with adverse maternal outcomes such as the increased risk of gestational diabetes mellitus, preeclampsia, caesarean-section delivery and bacterial vaginosis [32].

Randomized trials suggest that vitamin D supplementation can improve mood and health status in women. 8 The current study gives the conclusion that vitamin d deficiency could be associated with depression in women.

\section{Recommendations}

- Screening of vitamin d level especially for pregnant women due to its importance for mother and newborn, by making it a part of antenatal screening tests.

- The role of primary health care in the education of people particularly, pregnant women and the elderly about vitamin $d$ importance and the necessity of walking in sunshine in the early morning or afternoon for ultraviolet exposure.

- There is a need for more studies or surveys with the involvement of a larger sample as the current study focuses on basic things.

- Fortification of food with vitamin d especially the essential nutrient for children.

\section{Limitation of the Study}

Vitamin d deficiency is assessed in this study and the other studies in the world by depending on (RDA) system which means the Recommended Dietary Allowance for adults as Institute of Medicine estimated in 2011 that RDA is the appropriate level or the required supplementation of vitamin $\mathrm{d}$. This system fails to achieve serum concentrations above $20 \mathrm{ng} / \mathrm{ml}$ in some study participants due to the concept that the biologic reality of individual needs for any nutrient varies from person to person, generally in a normal distribution across the population.

Such conclusions, however, are based on misinterpretation and misapplication of the IOM reference values for vitamin D making the RDA unit controversial [22].

Serum vitamin d level is assessed by using $25(\mathrm{OH})$ vitamin D ELISA kit which is available in most labs in Iraq, but for a more accurate level, a storage form of 250HD could be used by depending an isotope dilution liquid chromatography- tandem mass spectrometry [11].

\section{Ethical Approval and Consent}

Data forms were anonymous. Confidentiality and privacy were assured. The collected data were used for research purposes only. Women's permission was taken verbally to publish this study for scientific purposes.

\section{Conflict of Interest: None}

\section{Funding: None}

\section{References}

1. Sizar 0, Givler A (2019) Vitamin D Deficiency. StatPearls Publishing. 


\section{Journal of Quality in Health Care \& Economics}

2. (2017) Vitamins and minerals. NHS. Overview.

3. Valle H Del, Yaktine A, Taylor C, Ross A (2012) Dietary Reference Intakes for Calcium and Vitamin D. Pediatrics 130(5): e1424-e1424.

4. Gowda U, Mutowo MP, Smith BJ, Wluka AE, Renzaho AMN (2015) Vitamin D supplementation to reduce depression in adults: Meta-analysis of randomized controlled trials. Nutrition 31(3): 421-429.

5. WHO (2017) Depression and Other Common Mental Disorders: Global Health Estimates pp: 24.

6. Parker GB, Brotchie H, Graham RK (2017) Vitamin D and depression. J Affect Disord 208: 56-61.

7. Penckofer S, Byrn M, Adams W, Emanuele MA, Mumby P, et al. (2017) Vitamin D Supplementation Improves Mood in Women with Type 2 Diabetes. J Diabetes Res 2017: 8232863.

8. Okereke OI, Singh A (2016) The role of Vitamin D in the prevention of late-life depression. J Affect Disord 198: 1-14.

9. Wang J, Li H, He Q, Ren C (2009) Effects of calcium and vitamin D supplementation on bone specific alkaline during pregnancy lactation and infant. Wei Sheng Yan Jiu 38(2): 193-195.

10. Marya RK, Rathee S, Manrow M (1987) Effect of calcium and vitamin D supplementation on toxaemia of pregnancy. Gynecol Obstet Invest 24(1): 38-42.

11. Vinkhuyzen AAE, Eyles DW, Burne THJ, Blanken LME, Kruithof CJ, et al. (2018) Gestational Vitamin D deficiency and autism-related traits: The Generation R Study. Mol Psychiatry 23(2): 240-246.

12. Lee SH, Ripke S, Neale BM, Faraone SV, Purcell SM, et al. (2013) Genetic relationship between five psychiatric disorders estimated from genome-wide SNPs. Nat Genet 45(9): 984-994.

13. Latimer CS, Brewer LD, Searcy JL, Chen KC, Popovic J KS et al. (2014) Vitamin D prevents cognitive decline and enhances hippocampal synaptic function in aging rats. Proc Natl Acad Sci USA 111: E4359-E4366.

14. Kaneko I, Sabir MS, Dussik CM, Whitfield GK, Karrys A, et al. (2015) 1,25-Dihydroxyvitamin D regulates expression of the tryptophan hydroxylase 2 and leptin genes: implication for behavioral influences of vitamin D. FASEB J 29(9): 4023-4035.
15. Keane VO, Marsh MS (2007) Depression during pregnancy Practice Depression during pregnancy. Small 334: 10-13.

16. Bennett HA, Einarson A, Taddio A, Koren G, Einarson TR (2004) Prevalence of depression during pregnancy: Systematic review. Obstet Gynecol 103(4): 698-709.

17. Lewis G, Ioannidis K, van Harmelen AL (2018) The association between pubertal status and depressive symptoms and diagnoses in adolescent females: A population-based cohort study. PLoS One 13(6): e0198804.

18. (2011) Harvard Health. Women and depressionHarvard Health.

19. Albert PR (2015) Why is depression more prevalent in women?. J Psychiatry Neurosci 40(4): 219-221.

20. Khoraminya N, Tehrani Doost M, Jazayeri S, Hosseini A, Djazayery A (2013) Therapeutic effects of vitamin D as adjunctive therapy to fluoxetine in patients with major depressive disorder. Aust NZJ Psychiatry 47(3): 271-275.

21. Mozaffari Khosravi H, Nabizade L, Yassini Ardakani SM, Hadinedoushan H, Barzegar K (2013) The effect of 2 different single injections of high dose of vitamin D on improving the depression in depressed patients with vitamin D deficiency: A randomized clinical trial. J Clin Psychopharmacol 33(3): 378-385.

22. Manson JAE, Brannon PM, Rosen CJ, Taylor CL (2016) Vitamin D Deficiency-Is There Really a Pandemic?. N Engl J Med 375(19): 1817-1820.

23. Swanson JG (1994) Family Physicians' Approach to Psychotherapy and Counseling. Psychotherapy 40: 53-58.

24. (2019) Vitamin D Deficiency Statistics UK - The Case for Supplementing.

25. Osuafor C, Fan CW, MacMahon M, McGreevy C, McCarthy F (2016) The practice of Vitamin D deficiency assessment in a Geriatric Day Hospital. Ir Med J 109(4): 393.

26. Whitehouse AJ, Holt BJ, Serralha M, Holt PG, Kusel MM, et al. (2012) Maternal serum vitamin D levels during pregnancy and offspring neurocognitive development. Pediatrics 129(3): 485-493. 


\section{Journal of Quality in Health Care \& Economics}

27. Eyles DW, Smith S, Kinobe R, Hewison M, McGrath JJ (2005) Distribution of the vitamin D receptor and 1 alpha-hydroxylase in human brain. J Chem Neuroanat 29(1): 21-30.

28. Song C, Wang H (2011) Cytokines mediated inflammation and decreased neurogenesis in animal models of depression. Prog Neuropsychopharmacol Biol Psychiatry 35(3): 760-768.

29. Cuomo A, Giordano N, Goracci A, Fagiolini A (2017) Depression and Vitamin D Deficiency: Causality, Assessment, and Clinical Practice Implications. Neuropsychiatry (London) 07(05): 606-614.

30. Mora JR, Iwata M, Von AUN (2008) Vitamin effects on the immune system: vitamins $A$ and D take centre stage. Rev Immunol 8(9): 685-698.
31. Dawodu A, Saadi HF, Bekdache G, Javed Y, Altaye M, et al. (2013) Randomized controlled trial (RCT) of vitamin $\mathrm{D}$ supplementation in pregnancy in a population with endemic vitamin d deficiency. J Clin Endocrinol Metab 98(6): 2337-2346.

32. Chakhtoura M, Nassar A, Arabi A, Cooper C, Harvey N, et al. (2016) Effect of Vitamin D replacement on maternal and neonatal outcomes: A randomised controlled trial in pregnant women with hypovitaminosis D. A protocol. BMJ Open 6(3): e010818. 